\title{
Prioritising wheelchair services for children: a pilot discrete choice experiment to understand how child wheelchair users and their parents prioritise different attributes of wheelchair services
}

Nathan Bray ${ }^{1 *}$, Seow Tien Yeo ${ }^{1}$, Jane Noyes ${ }^{2}$, Nigel Harris ${ }^{3}$ and Rhiannon Tudor Edwards ${ }^{1}$

\begin{abstract}
Background: Approximately 95 million children worldwide are disabled; $10 \%$ use a wheelchair. In the UK, an estimated 770,000 children are disabled. National Health Service Wheelchair Services are the largest provider of wheelchairs in the UK; however, recent reports have highlighted issues with these services. This study explores the use of discrete choice experiment methods to inform wheelchair service provision for disabled children based on service user preferences. The aim was to explore how disabled children and their parents prioritise different attributes of wheelchair services. The secondary aims were to compare priorities between parents and disabled children and to explore marginal rate of substitution for incremental changes in attributes.
\end{abstract}

Methods: Discrete choice experiments are a method of attribute-based stated preference valuation used by health economists to understand how individuals prioritise different attributes of healthcare services and treatments. We conducted the first pilot discrete choice experiment to explore how disabled children (aged 11 to 18) and their parents prioritise different attributes of hypothetical wheelchair services. Eleven disabled children (aged 11 to 18) and 30 parents of disabled children completed eight pairwise choice tasks based on five service attributes: wheelchair assessment, cost contribution, training, delivery time and frequency of review. Data were analysed using conditional logistic regression. For each pairwise choice, the participants were asked to choose which service scenario (A or B) they preferred.

Results: Comprehensiveness of wheelchair assessment and wheelchair delivery time significantly $(P<0.05)$ affected service preferences of children ( $\beta$-coefficients $=1.43$ [95 \% bootstrapped $\mathrm{Cl}=1.42$ to 2.08] and -0.92 [95\% bootstrapped $\mathrm{Cl}=-1.41$ to -0.84 ], respectively) and parents ( $\beta$-coefficients $=1.53$ [95\% bootstrapped $\mathrm{Cl}=1.45$ to 2.16 ] and -1.37 [95 \% bootstrapped $\mathrm{Cl}=-1.99$ to -1.31 ], respectively). Parents were willing to contribute more financially to receive preferred services, although this was non-significant.

Conclusions: Both samples placed the greatest importance on holistic wheelchair assessments encompassing more than health. The National Health Service should consider using discrete choice experiment methods to examine wheelchair service preferences of disabled children (aged 11 and over) and their parents on a wider scale; however, care must be taken to ensure that this method is used appropriately.

Keywords: Discrete choice experiment, Health economics, Conjoint analysis, Childhood disability, Wheelchair, Assistive technology

\footnotetext{
* Correspondence: n.bray@bangor.ac.uk

${ }^{1}$ Centre for Health Economics and Medicines Evaluation, Bangor University,

Ardudwy, Normal Site, Bangor, Gwynedd LL57 2PZ, UK

Full list of author information is available at the end of the article
} 


\section{Background}

It is estimated that 95 million children worldwide have a disability, 13 million of which have a severe disability [1]. Approximately $10 \%$ of disabled people require a wheelchair to maintain mobility [2]. Independent mobility for disabled people and provision of equipment to facilitate this are considered human rights by the United Nations [3]. Without adequate wheelchair provision, many disabled people are caught in a cycle of poverty and deprivation, with reduced access to education, work and social facilities [4]. It is estimated that 20 million disabled people worldwide do not have access to appropriate wheelchair equipment to maintain mobility and independence [4].

In the UK, there are an estimated 770,000 disabled people under the age of 16 [5], approximately 70,000 of which have unmet mobility needs [6]. The provision of a wheelchair at the appropriate time can offer a range of holistic benefits for disabled children and young people, for instance, functional mobility improvement [7], psychosocial development [8], development of communication skills $[7,9,10]$, and increased independence $[7,11]$.

Common physical disabilities which lead to mobility impairment include cerebral palsy, muscular dystrophy, spinal muscular atrophy and spina bifida. Traditional medical definitions describe disability as a deficit in functional ability, sensation or capacity. This assumes that the causes of disability are therefore a direct result of individual abnormal physical, cognitive or sensory functioning, thus defining disability as a disadvantage and human diversity as a scale from normal to abnormal [12]. The social model of disability focusses on how social oppression and discrimination disable those with impairments. Impairment and disability are therefore defined as two separate concepts; impairment is a bodily state characterised by physical or cognitive malfunction, while disability is the disadvantage or restriction faced by people with impairments due to societal, organisational and/or institutional barriers [13]. Wheelchairs offer a means for people with physical disabilities to alleviate some of their mobility issues and therefore reduce their experience of disability.

In the UK, National Health Service (NHS) Wheelchair Services are the largest provider of wheelchairs to disabled children. However, a number of high-profile inquiries have highlighted inadequacies in NHS wheelchair services for children and young people [14-16]. The National Assembly for Wales Health, Wellbeing and Local Government Committee report [17] found that Welsh NHS wheelchair services were supplying inadequate wheelchair interventions to enable children to lead fulfilled lives. Service development recommendations included reduced waiting times; a more holistic approach to wheelchair provision (taking into account social, educational and developmental outcomes); and improvement of inadequate review procedures and information provision.

At present, there is no published evidence as to how wheelchair service users prioritise different attributes of wheelchair services either explicitly or implicitly; thus, the relative importance of these attributes to service users is not currently known. Disabled children and their parents should be engaged in shaping wheelchair services at the local level $[18,19]$. In order for this to be achieved, it is important to understand how service users prioritise the different attributes of wheelchair services.

\section{Aims and objectives}

The primary aim of this pilot study was to explore the preferences of disabled children and their parents for different attributes of wheelchair services. The secondary objectives were as follows:

- To compare the preferences of disabled children and their parents for different attributes of wheelchair services

- To calculate hypothetical marginal rate of substitution (MRS) values for different configurations of wheelchair services using cost contribution as the denominator

- To illustrate the use of discrete choice experiment (DCE) methods with disabled children, in relation to wheelchair services

\section{Methods}

\section{Ethical considerations}

The study was granted ethical approval by the North West Wales NHS Research Ethics Committee (reference: 13/WA/0143) and an academic ethics committee at Bangor University. Eligible participants were sent postal information about the study and indicated their consent to participate by returning a completed demographic questionnaire, after which a date and time for administration of the DCE questionnaire was arranged. The participants were offered a small financial incentive (a $£ 10$ retail voucher) for taking part in the study.

Before administering the DCE questionnaire the study was explained in full to the participants, and they were informed of the data collection process and the aims of the research. The participants were then asked to complete a second consent/assent form to indicate that they understood and agreed to take part. Children under the age of 16 completed an assent form, and their parents completed a proxy consent form. Data collection was conducted in the home of the participants where possible. 


\section{Sampling and recruitment}

A convenience sample was used. The sampling frame was disabled children (aged 18 or under) who use a wheelchair and their parents. The pilot DCE was part of a larger programme of research called the Wheels Project (funded by the National Institute for Social Care and Health Research), which also included assessment of health-related quality of life. This paper presents only the DCE data.

The participants were recruited between June and October 2013 from three recruitment sites: an NHS wheelchair service, a charity-powered wheelchair (PWC) manufacturer/supplier and a children's wheelchair charity. The DCE questionnaire was completed as part of a separate interview for the Wheels Project. One parent per child was asked to complete the DCE.

\section{DCE design}

DCEs are an established method of conjoint analysis used in health economics to elicit stated preferences for different services or different attributes of services. Individuals are asked to make decisions between hypothetical scenarios with differing attributes. Their preferences, and trade-offs between levels of attributes, are then inferred based on their patterns of choices. DCEs are therefore a form of attribute-based stated preference valuation. A DCE is designed as a number of hypothetical scenarios arranged into paired choice scenarios. These paired choice scenarios have a set number of attributes (e.g. cost) with varying levels (e.g. $£ 50$ or $£ 150$ ) chosen by the researcher based on previous research and expertise. Individuals are asked to make trade-offs between the attributes in the DCE tasks by comparing the variation of levels between pairwise choices and then choosing between the two or more competing hypothetical scenarios, thus revealing their relative preference for different service attributes [20]. The development and reporting of this DCE meets the criteria set out in the ISPOR conjoint analysis checklist [21].

The DCE attributes, levels and design for this pilot study were derived from a mixed-method systematic review of the literature [19] and through discussion with young wheelchair users (aged 11 to 18) and healthcare professionals working within wheelchair services. The systematic review [19] incorporated a range of mixedmethod evidence relating to wheelchair services and service users experiences of them. Government reports and policy evidence were thematically coded and synthesised to produce seven emergent categories of wheelchair service priority areas: waiting times; joint working and multi-agency approach; effective use and outcomes; funding and procurement; aftercare and information; eligibility criteria and assessment; and service user involvement. The overarching findings from the systematic review were synthesised to develop a conceptual framework for effective wheelchair service development, which was subsequently used to refine the emergent categories into ten wheelchair service attributes (and their levels) for the DCE.

A list of these ten attributes (and their levels) was presented to healthcare professionals in wheelchair provision $(n=5)$, who were asked to consider which five attributes they felt were most relevant to the current practice and which levels were most reflective of the current practice and service targets. As the DCE was intended to be completed by children, we chose to limit the design to five attributes in order to reduce burden on the respondents. Five attributes from the original list of ten were excluded following consensus from the wheelchair professionals: waiting time for assessment; distance of wheelchair service from patients' home; patient transport provided by wheelchair service; availability of loan equipment; and cost of maintenance. The remaining five key attributes were as follows: (1) comprehensiveness of wheelchair assessment, (2) cost contribution for wheelchair, (3) level of training provided by service, (4) waiting time for delivery of wheelchair and (5) frequency of wheelchair reviews. Of these five attributes, four were assigned two levels (e.g. wait 1-3 months or 6-12 months for delivery) and one was assigned four levels (e.g. pay nothing, £50, £150 or $£ 300$ ). See Table 1 for a full list of attributes, levels and effect codes. The attribute levels represent both the current NHS practice and aspirational practice:

- Holistic wheelchair assessments (considering important factors such as social and educational needs) have been recommended by a number of government reports, as clinical need has historically been solely used to assess assistive technology needs $[14,17]$.

- The average cost of a standard manual wheelchair supplied by the NHS is $£ 270$ [22]. The NHS is free at the point of care; however, service users can choose to purchase wheelchairs privately through the NHS wheelchair voucher scheme, which allows service users to request a voucher towards the cost of a privately funded wheelchair [23]. The attribute levels were set relatively low in order to reflect a non-prohibitive service user contribution.

- NHS services provide wheelchair skills training as part of standard practice, while charitable organisations, such as Whizz-Kidz, provide advanced wheelchair skills training and life skills training outside of the NHS.

- Delivery time for NHS wheelchairs varies across services, with children often waiting up to a year for a wheelchair [17]; the aspirational target is less than 18 weeks from referral to delivery [24]. 
Table 1 Full list of attributes, levels and effect codes for discrete choice experiment questionnaire (child version)

\begin{tabular}{|c|c|c|}
\hline Attribute & Level & Definition (effect coding) \\
\hline \multirow{2}{*}{$\begin{array}{l}\text { Comprehensiveness of wheelchair } \\
\text { assessment }\end{array}$} & Health needs & Your health needs will be considered in the wheelchair assessment (0) \\
\hline & $\begin{array}{l}\text { Health, school and } \\
\text { social life needs }\end{array}$ & $\begin{array}{l}\text { Your health, school and social life needs will be considered in the } \\
\text { wheelchair assessment (1) }\end{array}$ \\
\hline \multirow[t]{4}{*}{ Cost $(£)$ contribution for wheelchair } & No cost & You will not have to contribute any money for your wheelchair (0) \\
\hline & $£ 50$ & $\begin{array}{l}\text { You will have to contribute } £ 50 \text { for your wheelchair. This would be a } \\
\text { one-off payment for each new wheelchair ( } 50 \text { ) }\end{array}$ \\
\hline & $£ 150$ & $\begin{array}{l}\text { You will have to contribute } £ 150 \text { for your wheelchair. This would be a } \\
\text { one-off payment for each new wheelchair (150) }\end{array}$ \\
\hline & $£ 300$ & $\begin{array}{l}\text { You will have to contribute } £ 300 \text { for your wheelchair. This would be a } \\
\text { one-off payment for each new wheelchair ( } 300)\end{array}$ \\
\hline \multirow[t]{2}{*}{ Level of training provided by service } & Wheelchair skills training & $\begin{array}{l}\text { You will receive wheelchair skills training as part of the service. Wheelchair } \\
\text { skills training will include wheelchair driving techniques, road safety and } \\
\text { maintaining your wheelchair (0) }\end{array}$ \\
\hline & $\begin{array}{l}\text { Wheelchair and life skills } \\
\text { training }\end{array}$ & $\begin{array}{l}\text { You will receive wheelchair skills training and life skills training as part of the } \\
\text { service. Wheelchair skills training will include wheelchair driving techniques, } \\
\text { road safety and maintaining your wheelchair. Life skills training will include } \\
\text { work placements, learning independence and ambassador groups (1) }\end{array}$ \\
\hline \multirow[t]{2}{*}{ Delivery time for delivery wheelchair } & Between 1 and 3 months & $\begin{array}{l}\text { It will take between } 1 \text { and } 3 \text { months for your wheelchair to be delivered after } \\
\text { the final assessment (0) }\end{array}$ \\
\hline & Between 6 and 12 months & $\begin{array}{l}\text { It will take between } 6 \text { and } 12 \text { months for your wheelchair to be delivered after } \\
\text { the final assessment (1) }\end{array}$ \\
\hline \multirow[t]{2}{*}{ Frequency of wheelchair review } & At least every 6 months & $\begin{array}{l}\text { Your needs and wheelchair will be reviewed every } 6 \text { months. This will include } \\
\text { a reassessment of your needs and a review of your wheelchair for any } \\
\text { maintenance or repairs it requires (6) }\end{array}$ \\
\hline & At least every 12 months & $\begin{array}{l}\text { Your needs and wheelchair will be reviewed every } 12 \text { months. This will } \\
\text { include a reassessment of your needs and a review of your wheelchair } \\
\text { for any maintenance or repairs it requires (12) }\end{array}$ \\
\hline
\end{tabular}

- NHS services aim for wheelchair reviews and maintenance to be undertaken at least annually [25], although more frequent reviews and maintenance could be beneficial [24].

Following feedback from wheelchair professionals, a preliminary DCE was designed and presented to a small sample $(n=10)$ of young wheelchair users (aged 11 to 18) at a children's wheelchair charity beneficiary meeting in order to gauge their understanding of the DCE method and the appropriateness of the attributes, levels and questionnaire design.

Subsequent to the feedback obtained from the beneficiary meeting, the design and layout of the DCE was refined in order to make it easier to understand for children from age 11. This included developing pictorial representations of the attributes and levels to increase ease of use (see Fig. 1). No further changes to the attributes and levels were recommended by the young wheelchair users. Two versions of the DCE questionnaire were developed to allow for slight differences in wording of questions for parents and children, although the content of the tasks remained the same.

The final combination of attributes and levels produced a full factorial design of 64 hypothetical service scenarios. For ease of completion, an appropriate mixedlevel orthogonal array was used to reduce the number of scenarios down to eight with efficient design [26]. Coding of attribute levels for the eight scenarios was obtained from an appropriate mixed-level orthogonal array [26]. Scenarios were mirrored and paired in a fold-over design, so that each of the eight scenarios had a mirrored alternative with opposite attribute levels, giving a total of 16 scenarios in eight pairwise choice tasks. This ensured that there was minimum overlap and attribute levels were not repeated across pairwise choices. For each pairwise choice, the participants were asked to choose which of the two hypothetical service scenarios (service A or service B) they preferred. See Table 2 for an example of a pairwise choice task.

Instructions on how to complete the DCE questionnaire were presented to the participants at the beginning of the questionnaire. A supplementary notes section was included with the questionnaire for further information on the attributes and levels (see Additional file 1). In most cases, a researcher was present during completion of the DCE to answer any questions. A small number of participants $(n=6)$ were sent the DCE questionnaire via post; therefore, they completed the questionnaire without a researcher present. Subsequent sub-group analysis 


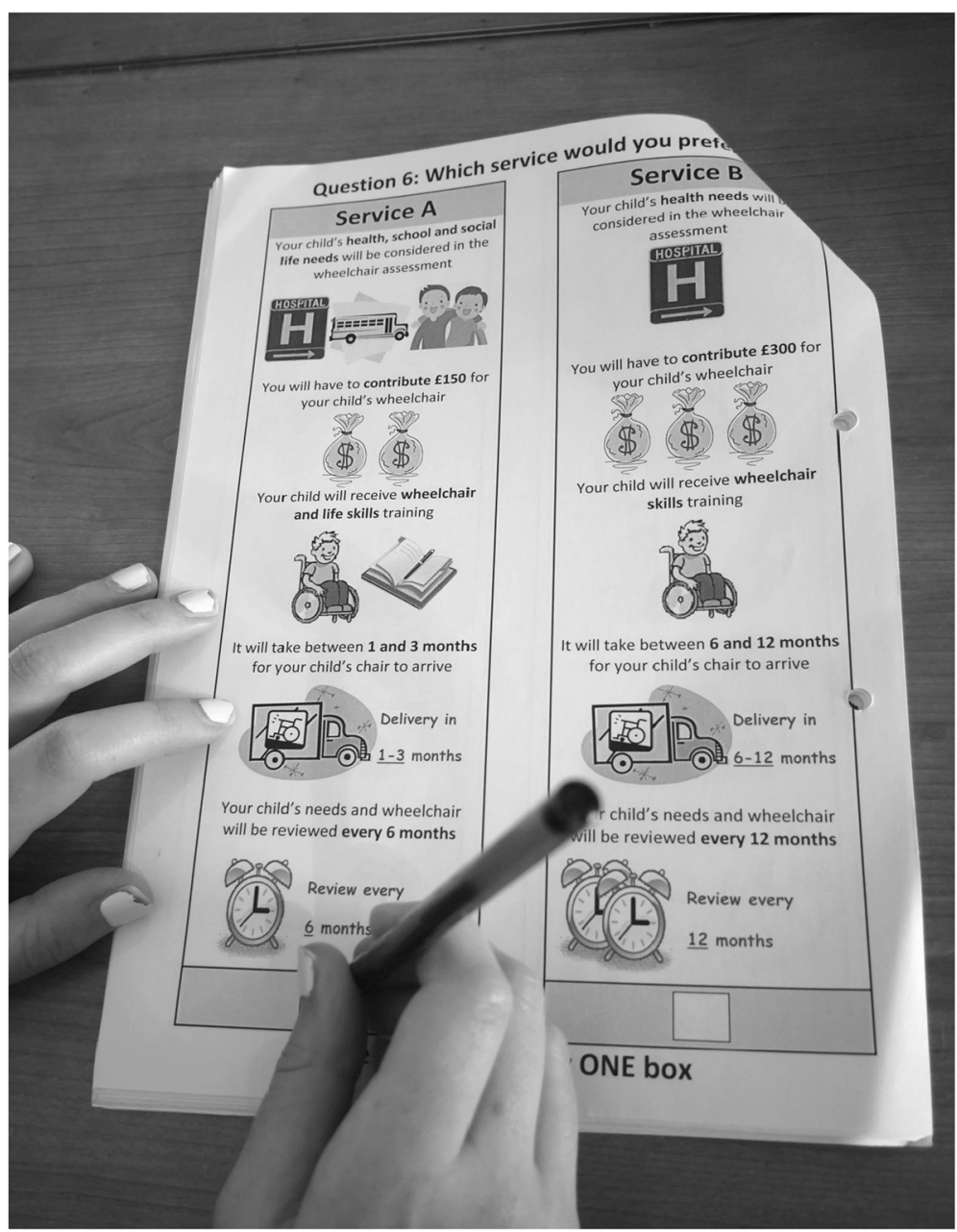

Fig. 1 Example of parent DCE questionnaire being completed. Pictorial representations of the DCE attribute and levels, as displayed in the DCE questionnaires completed by children and their parents

Table 2 Example of parent DCE pairwise choice task

\begin{tabular}{ll}
\hline Service A & Service B \\
\hline $\begin{array}{l}\text { Your child's health, school and social life needs will be considered } \\
\text { in the wheelchair assessment }\end{array}$ & $\begin{array}{l}\text { Your child's health needs will be consid } \\
\text { assessment } \\
\text { The service will be free }\end{array}$ \\
$\begin{array}{l}\text { You will have to contribute } £ 50 \text { for your } \\
\text { Your child will receive wheelchair skills trair receive wheelchair and life skills training }\end{array}$ & $\begin{array}{l}\text { It will take between } 1 \text { and } 3 \text { months for } \\
\text { It will take between } 6 \text { and } 12 \text { months for your child's chair to arrive }\end{array}$ \\
Your child's needs and wheelchair will be reviewed every 6 months & Your child's needs and wheelchair will bervice would you prefer? Please tick one box below: \\
\end{tabular}


to examine the effect of researcher presence was not carried out due to the small sub-group size.

\section{Data analysis}

SPSS v20.0 (IBM Corp., Armonk, New York, USA) and Stata v10.1 (StataCorp, College Station, Texas, USA) were used to analyse the data. In the main analyses, the child and parent samples were analysed separately. Data were analysed using logistic regression; the conditional logistic regression model was used [27, 28]. For each given pairwise choice, "1" represents service A chosen, and " 0 " represents service B chosen. Fixed effects logit modelling was used to analyse these data, with choice of service scenario as the dependent variable. The model represents the utility function of how the analysed sample trade-off between service attributes, by selecting either scenario (A or B) in each given pairwise choice set.

The statistical model presented in Additional file 2 shows the equation that describes the estimated fixed effects conditional logistic regression model. In the model, utility is assumed linear and additive [28]. The model represents a linear in parameters main effects utility function, a classic functional form used widely in previous DCEs [29-31]. The deterministic component of the utility function ( $\Delta$ utility) is a function of the attribute levels between service scenarios, where the coefficients of each attribute and the constant term are estimated in the model. The $\beta$-coefficients are subsequently summed to estimate utility for each combination of attribute levels. This gives an indication of the relative social value of each potential service scenario, and the potential impact of individual attribute levels.

The model enables an analyst to interpret the output of the regression model to determine three factors; firstly, the importance of attributes, assessed by coefficient significance levels (i.e. $P$ value less than 0.05 ); secondly, the effect of an attribute on utility, assessed by examining the $\beta$-coefficient of each attribute in which the $\beta$-coefficient shows change in utility when moving from one attribute's level to another attribute's level; and finally, the MRS, used to examine the trade-offs between attributes. Clustering of responses was not included in the model due to the small sample size. Clustering would need to be considered in a full DCE in this population.

Using this model, the magnitude of the $\beta$-coefficient is relative to the change in utility as a result of change in the attribute's level. A positive $\beta$-coefficient indicates that as the level increases, so does the likelihood of a participant choosing it. Likewise, a negative $\beta$-coefficient indicates that as the level increases, the likelihood of participants choosing it decreases.

We hypothesised that a positive $\beta$-coefficient would be observed for the comprehensiveness of wheelchair assessment attribute and level of training provided by service attribute, as the participants were expected to prefer to have additional in-depth assessment of needs and additional training. For the other attributes, it was hypothesised that a negative $\beta$-coefficient would be observed, as the participants were expected to prefer lower cost contribution, shorter waiting time for delivery and more regular wheelchair reviews.

Effects codes were assigned to the attribute levels of all quantitative (continuous) and qualitative (non-continuous) variables in the DCE design. All of the qualitative variables were dichotomous; thus, the effect codes of 0 and 1 were assigned to their levels as appropriate. The difference between the effect codes of a qualitative attribute (when moving from "alternative A" to "alternative $B$ " in each pairwise choice) was therefore calculated as either -1 or +1 and assigned to the corresponding qualitative attribute in the Stata dataset.

Attributes were not directly comparable on the same scale due to a mix of quantitative and qualitative attributes; thus, MRS was calculated to attain common scale for all attributes. This allowed comparison between attributes to be made. MRS is the amount of a given attribute that a person is willing to forgo in order to obtain one additional unit of another attribute. For instance, an individual may be willing to contribute towards the cost of the wheelchair in order to reduce the delay in wheelchair delivery. The cost contribution attribute (quantitatively scaled) was used as the denominator to calculate the MRS for a one-unit change in each of the remaining attributes. By dividing the other attribute coefficients by the cost contribution coefficient, the MRS was indirectly estimated. The $95 \%$ confidence intervals for the $\beta$-coefficients were estimated using non-parametric bootstrapping methods, ran on 5000 iterations using Stata v10.1. All presented confidence intervals are therefore bootstrapped.

\section{Implementation \\ Sample and response rates}

A total of 125 study invitation packs were distributed across England and Wales by the three recruitment sites. These contained initial questionnaires for parents and disabled children. Parents and disabled children were recruited from the same household where possible and appropriate. Thirty-five initial questionnaires were returned by the parents ( $28 \%$ response rate), who were then invited to complete the DCE questionnaire. Of that number, 30 parent DCE questionnaires were completed in full $(85.7 \%$ response rate).

Of the disabled children who returned an initial questionnaire $(n=15), 13$ met eligibility criteria (aged $>10)$ and were invited to complete the DCE questionnaire. A total of 11 disabled children completed the DCE questionnaire (84.6\% response rate). 
All returned DCE questionnaires were completed in full with no major data omissions.

\section{Demographic characteristics}

Demographic details of the samples are presented in Tables 3 and 4. In the disabled child sample, $63.6 \%(n=7)$ were male, $63.6 \%(n=7)$ were aged 16 to 18 and $81.8 \%$ $(n=9)$ had cerebral palsy. In the parent sample, $86.7 \%$ $(n=26)$ of the respondents were women, $86.7 \%(n=26)$ were aged between 30 and 49 and $66.7 \%(n=20)$ had a child with cerebral palsy. There is a lack of ethnic diversity in both samples with the vast majority of respondents being white British.

\section{Results}

\section{DCE results: disabled child sample}

Table 5 shows the results for the two samples. The disabled child sample results show that the $\beta$-coefficients of three of the five attributes were statistically significant

Table 3 Demographic characteristics of the disabled child sample $(n=11)$

\begin{tabular}{ll}
\hline Demographic characteristics & Number (\%) \\
\hline Study site & $2(18.2)$ \\
NHS wheelchair service & $9(81.8)$ \\
Children's wheelchair charity & \\
Gender & $4(36.4)$ \\
Female & $7(63.6)$ \\
Male & \\
Age & $4(36.4)$ \\
11-15 years & $7(63.6)$ \\
16-18 years & \\
Ethnicity & $11(100)$ \\
White British & \\
Education & $4(36.4)$ \\
High school & $5(45.5)$ \\
College & $1(9.1)$ \\
University & $1(9.1)$ \\
Home schooled & \\
Child's condition & \\
Cerebral palsy & \\
Muscular dystrophy & \\
Hemiplegia/stroke & $9(81.8)$ \\
Frequency of equipment use & $1(9.1)$ \\
All of the time & $1(9.1)$ \\
Mype of equipment used & \\
Manual & $10(90.9)$ \\
\hline
\end{tabular}

$(P<0.05)$ : comprehensiveness of wheelchair assessment $(\beta$-coefficient $=1.4247[P=0.009 ; 95 \%$ bootstrapped $\mathrm{CI}$ $=1.42$ to 2.08$]$ ), waiting time for delivery of wheelchair $(\beta$-coefficient $=-0.9221[P=0.041 ; 95 \%$ bootstrapped $\mathrm{CI}=-1.41$ to -0.84$])$ and cost contribution for wheelchair $(\beta$-coefficient $=-0.0093 \quad[P=0.019 ; 95 \%$ bootstrapped $\mathrm{CI}=-0.014$ to -0.009$])$. The remaining two attributes were non-significant: level of training provided by service $(\beta$-coefficient $=0.0306[P=0.924 ; 95 \%$ bootstrapped $C I=-0.20$ to 0.29$])$ and frequency of wheelchair reviews $(\beta$-coefficient $=0.0364 \quad[P=0.519 ; 95 \%$ bootstrapped $\mathrm{CI}=0.00$ to 0.08$]$ ). Of the attributes that were significant, comprehensiveness of wheelchair assessment was of greatest importance $(\beta$-coefficient $=$ $1.4247)$, followed by waiting time for delivery of wheelchair $(\beta$-coefficient $=-0.9221)$ and cost contribution for wheelchair $(\beta$-coefficient $=-0.0093)$. Preference was shown for comprehensive wheelchair assessments (of health, education and social needs), shorter waiting time for delivery of wheelchair and lower cost contribution. For the remaining two non-significant attributes, disabled children preferred (if everything being equal) wheelchair and life skills training and less frequent wheelchair reviews.

\section{DCE results: parent sample}

The parent sample results show that the $\beta$-coefficients of two of the five attributes were statistically significant $(P<0.05)$. These were comprehensiveness of wheelchair assessments $\quad(\beta$-coefficient $=1.5329 \quad[P<0.0001 ; 95 \%$ bootstrapped $\mathrm{CI}=1.45$ to 2.16$]$ ) and waiting time for wheelchair delivery $(\beta$-coefficient $=-1.3699[P<0.0001$; $95 \%$ bootstrapped $\mathrm{CI}=-1.99$ to -1.31$]$ ). This indicates that these two attributes were significant factors in parental choices. The remaining three attributes were nonsignificant: cost contribution for wheelchair $(\beta$-coefficient $=-0.0028[P=0.092 ; 95 \%$ bootstrapped $\mathrm{CI}=-0.01$ to 0.00$])$, level of training provided by service $(\beta$-coefficient $=-0.1557[P=0.371 ; 95 \%$ bootstrapped $\mathrm{CI}=-0.40$ to 0.03$])$ and frequency of wheelchair reviews $(\beta$-coefficient $=-0.0390[P=0.260 ; 95 \%$ bootstrapped $\mathrm{CI}=-0.08$ to 0.00$])$. Based on the $\beta$-coefficients of the significant attributes, comprehensiveness of wheelchair assessment was of greatest importance $(\beta$-coefficient $=1.5329)$, followed by waiting time for delivery of wheelchair $(\beta$ coefficient $=-1.3699$ ). Preference was shown for comprehensive wheelchair assessments (of health, education and social needs) and shorter waiting time for delivery of wheelchair. For the remaining three non-significant attributes, parents preferred (if everything being equal) lower cost contribution, basic wheelchair skills training and more frequent wheelchair reviews. As cost contribution was not significant, parental MRS values are not reliable. 
Table 4 Demographic characteristics of the parent sample $(n=30)$

\begin{tabular}{l} 
Demographic characteristics \\
\hline Study site \\
NHS wheelchair service \\
Charity PWC supplier \\
Children's wheelchair charity \\
Gender \\
Female \\
Male \\
Age \\
21-29 years \\
30-39 years \\
40-49 years \\
50-59 years \\
Ethnicity
\end{tabular}

\section{White British}

White and Asian

Marital status

Married

Co-habiting

Single

Separated

Divorced

Annual household Income

$£ 5000-15,000$
$£ 16,000-£ 25,000$
$£ 26,000-£ 35,000$
$£ 36,000-£ 50,000$
$£ 51,000$ or more
Missing

Employment status

Full-time

Part-time

Unemployed/stay-at-home parent

Child's condition/disability

Cerebral palsy

Spinal muscular atrophy

Muscular dystrophy

Others

Child's age

5 years or under

6-15 years

16-18 years

Frequency of child's equipment use

A little of time

Some of the time
Number (\%)

\section{5 (16.7)}

$10(33.3)$

$15(50.0)$

$26(86.7)$

$4(13.3)$

$2(6.7)$

$14(46.7)$

$12(40.0)$

$2(6.7)$

$29(96.7)$

1 (3.3)

$23(76.7)$

$3(10.0)$

$2(6.7)$

1 (3.3)

$1(3.3)$

$3(10.0)$

5 (16.7)

$3(10.0)$

$10(33.3)$

8 (26.6)

1 (3.3)

$5(16.7)$

$12(40.0)$

$13(43.3)$

$20(66.7)$

$2(6.7)$

$3(10.0)$

$5(16.5)$

15 (50.0)

10 (33.3)

5 (16.7)

1 (3.3)

$6(20.0)$
Table 4 Demographic characteristics of the parent sample $(n=30)$ (Continued)

\begin{tabular}{ll}
\hline Most of the time & $4(13.3)$ \\
All of the time & $18(60.0)$ \\
Missing & $1(3.3)$ \\
Type of equipment used by child & \\
Powered & $2(6.7)$ \\
Manual & $10(33.3)$ \\
Manual and powered & $17(56.7)$ \\
Waiting for first wheelchair & $1(3.3)$ \\
\hline
\end{tabular}

\section{Comparison of disabled child and parent DCE and MRS} results

Both samples showed preference for comprehensive wheelchair assessments and shorter wheelchair delivery times, in that order. The cost contribution attribute was only significant for the child sample, who showed preference for lower cost contribution. Although cost contribution was not significant for parents, for comparison purposes, we calculated the parental MRS values. Results show that the MRS values were higher for parents ( $£ 547.46$ [95 \% bootstrapped $\mathrm{CI}=£ 353.38$ to $£ 1435.45$ ] for wheelchair assessment and $£ 489.25$ [95 \% bootstrapped $\mathrm{CI}=£ 313.29$ to $£ 1326.77]$ for delivery waiting time) than for disabled children ( $£ 153.19$ [95 \% bootstrapped CI $=£ 133.20$ to $£ 182.53]$ for wheelchair assessment and $£ 99.15$ [95 \% bootstrapped CI $=£ 81.93$ to $£ 121.32]$ for delivery waiting time), suggesting the parent sample placed higher importance on these attributes than the disabled child sample. However, as the cost contribution attribute was not significant for parents, it is difficult to make comparisons with the disabled child data.

The disabled child and parent samples differed in direction of coefficient preference for level of training provided by service and frequency of wheelchair reviews: the $\beta$-coefficients for both attributes indicate that, everything being equal, parents preferred basic wheelchair skills training $(\beta$-coefficient $=-0.1557)$ and more frequent wheelchair reviews $(\beta$-coefficient $=$ $-0.0390)$, while disabled children preferred wheelchair and life skills training $(\beta$-coefficient $=0.0306)$ and less frequent wheelchair reviews $(\beta$-coefficient $=0.0364)$. However, the $\beta$-coefficients for these attributes were not significant.

\section{Sub-group analysis: matched pairs of disabled children and their parents}

In order to compare preferences of disabled children and their respective parents, a sub-group analysis was performed using only the data from dyads of disabled children $(n=9)$ and their respective parents $(n=9)$, see 
Table 5 Results from conditional logistic regression model: disabled child study sample $(n=11)$ and parent study sample $(n=30)$

\begin{tabular}{|c|c|c|c|c|c|c|c|c|c|c|}
\hline \multirow[t]{2}{*}{ Attribute } & \multicolumn{5}{|c|}{ Disabled child sample $(n=11)$} & \multicolumn{5}{|c|}{ Parent sample $(n=30)$} \\
\hline & $\overline{\beta \text {-coefficient }}$ & $\begin{array}{l}95 \% \text { bootstrapped } \\
\mathrm{Cl}^{\mathrm{a}}\end{array}$ & $P$ value & $\begin{array}{l}\text { MRS values }^{b} \\
\text { (cost) }\end{array}$ & $\begin{array}{l}95 \% \text { bootstrapped } \\
\mathrm{Cl}^{\mathrm{a}}\end{array}$ & $\bar{\beta}$-coefficient & $\begin{array}{l}95 \% \text { bootstrapped } \\
\mathrm{Cl}^{\mathrm{a}}\end{array}$ & $P$ value & $\begin{array}{l}\text { MRS values, } \\
\text { (cost) }\end{array}$ & $\begin{array}{l}95 \% \text { bootstrapped } \\
\mathrm{Cl}^{\mathrm{a}}\end{array}$ \\
\hline $\begin{array}{l}\text { Comprehensiveness of wheelchair } \\
\text { assessment }\end{array}$ & $1.4247^{*}$ & 1.4153 to 2.0824 & 0.009 & $£ 153.19$ & $£ 133.20$ to $£ 182.53$ & $1.5329^{*}$ & 1.4507 to 2.1633 & $<0.0001$ & $£ 547.46$ & $£ 353.38$ to $£ 1435.45$ \\
\hline Cost contribution for wheelchair & $-0.0093^{*}$ & -0.0138 to -0.0089 & 0.019 & - & - & -0.0028 & -0.0060 to 0.0005 & 0.092 & - & - \\
\hline Level of training provided by service & 0.0306 & -0.1955 to 0.2858 & 0.924 & - & - & -0.1557 & -0.4002 to 0.0311 & 0.371 & - & - \\
\hline Waiting time for delivery of wheelchair & $-0.9221^{*}$ & -1.4086 to -0.8442 & 0.041 & $£ 99.15$ & $£ 81.93$ to $£ 121.32$ & $-1.3699^{*}$ & -1.9859 to -1.3104 & 0.000 & $£ 489.25$ & $£ 313.29$ to $£ 1326.78$ \\
\hline Frequency of wheelchair reviews & 0.0364 & -0.0022 to 0.0749 & 0.519 & - & - & -0.0390 & -0.0813 to 0.0032 & $<0.0001$ & - & - \\
\hline \multicolumn{6}{|l|}{ Number of observations $=88$} & \multicolumn{5}{|c|}{ Number of observations $=240$} \\
\hline \multicolumn{6}{|l|}{ Number of individuals $=11$} & \multicolumn{5}{|c|}{ Number of individuals $=30$} \\
\hline \multicolumn{6}{|l|}{ Log likelihood function $=-26.64$} & \multicolumn{5}{|c|}{ Log likelihood function $=-64.51$} \\
\hline \multicolumn{6}{|l|}{ Log likelihood ratio (5) $=33.85$} & \multicolumn{5}{|c|}{ Log likelihood ratio $(5)=114.86$} \\
\hline
\end{tabular}

*Significant attribute $[P<0.05]$

${ }^{a} 95 \%$ confidence intervals generated using non-parametric bootstrapping (5000 replications)

${ }^{\mathrm{b}}$ Marginal rate of substitution values $=\beta$-coefficient for attribute $/ \beta$-coefficient for cost attribute

'Though the cost contribution attribute was not significant to parents $(P=0.092[>0.05])$, everything being equal, parents preferred lower cost contribution; the parents' MRS values were calculated using the cost

contribution attribute as the denominator to show how parents trade-off the cost contribution attribute against the other attributes. This allowed comparison with the disabled child sample MRS values 
Additional file 3. Analyses were conducted separately, and preferences were then compared between related disabled children and parents. A smaller distribution of child age was observed, with all children aged 11 or over (63.6 \% [ $n=7$ ] aged 16 or over).

Similarly to the main analysis, both the disabled child and parent samples showed significant preference for comprehensive wheelchair assessments: $\beta$-coefficients equalled 1.6194 $(P=0.015 ; 95 \%$ bootstrapped $\mathrm{CI}=1.58$ to 2.29$)$ and $2.1893(P=0.010 ; 95 \%$ bootstrapped $\mathrm{CI}=$ 2.21 to 3.26) respectively. The cost contribution attribute was again not significant for the parent sample but was borderline significant for the child sample, who showed preference for lower cost contribution $(\beta$-coefficient $=-0.0095[P=0.050 ; 95 \%$ bootstrapped $\mathrm{CI}=-0.013$ to -0.009$])$. All other attributes were non-significant.

\section{Discussion}

This is the first study to systematically elicit and compare the preferences of disabled children and their parents for different attributes of wheelchair services. The findings illustrate the use of the DCE method in a potentially vulnerable sample of the population for the assessment of healthcare services. If appropriately powered, DCE results can be used as a valuable asset in priority setting, service development and healthcare decision-making, as they allow different attributes of services to be ranked by importance, and their relative monetary value to families calculated using MRS.

For the sample of 11 disabled children and 30 parents of disabled children, comprehensiveness of wheelchair assessment was the most important attribute of wheelchair services, followed by wheelchair delivery time. The results from the disabled child sample indicated that cost contribution was also influential. The remaining two attributes (level of training provided by service and frequency of wheelchair reviews) were not statistically significant $(P>0.05)$ for both samples, and thus, they did not impact service preferences.

Both samples showed preference for services that offered assessments which focused on the health, education and social needs of children, as opposed to just health needs. NHS wheelchair services tend to focus on clinical health needs in wheelchair assessment and provision, which may neglect to consider other important aspects of disabled children's lives [17].

The MRS values of the wheelchair assessment and delivery time attributes were different for the two samples, with parental MRS values higher for both attributes. This would suggest that the sampled parents were willing to contribute more money to attain preferred service attributes. However, it is important to reiterate that cost contribution was not a significant attribute for the sampled parents, while it was for the sampled disabled children. This may reflect that the parent sample were willing to pay more to obtain the best suited services for their child, thus contribution cost did not influence their preferences. Assumptions regarding MRS should be viewed with caution as adults and children are likely to value money differently.

Identical cost contribution levels were used for both samples, which did not take into account differences in how the two samples value money, particularly as the sampled disabled children would have expected to spend family money rather than their own. In hindsight, sampled parents may have felt that contributing up to $£ 300$ for a wheelchair was relatively good value for money compared to purchasing a wheelchair privately, while sampled disabled children may have considered this to be a significant amount of money. It is of note that $60 \%$ $(n=18)$ of sampled parents had a household income of over $£ 36,000$ per year, which may have impacted their willingness to contribute financially to receive a preferred services for their child.

A future DCE in this field should consider using considerably higher cost contribution attribute levels to test these issues, for instance, setting the levels at retail prices for different types of wheelchairs (e.g. $£ 500$, $£ 1500, £ 3000$ ), or conversely using a more child-friendly approach to cost contribution, such as proportion of income/pocket money. A larger sample would be needed to enable additional sub-group analyses, such as analysing the effect of household income on preferences and MRS values.

Most of the attribute $\beta$-coefficient directions are reflective of a priori hypotheses, although the coefficient directions for frequency of wheelchair reviews for the disabled child sample and level of training for the parent sample were contradictory to these hypotheses. Although not statistically significant, the coefficient direction for the level of training attribute may indicate that sampled parents did not feel it was the responsibility of wheelchair services to provide life skills alongside wheelchair skills training, or potentially that the provision of life skills training may impact on essential wheelchair skills training. This may be a result of the parent sample being skewed towards parents of younger children, as life skills training is likely to be of most importance to older children who want to develop independence. Future research may benefit from defining life skills training based on age (e.g. play skills for children under 5).

For the frequency of wheelchair reviews attribute, the disabled child sample preferred less frequent reviews, although this was also non-significant. This may indicate that the sampled disabled children did not necessarily see the benefit of more frequent reviews of their needs or they may not enjoy reviews and thus would prefer them to be less frequent. 
Comparing the results of the full and sub-sample analyses shows variation in parental preferences for frequency of wheelchair reviews and the significance of delivery time on service preference. The parent subsample preferred less frequent reviews (as did their children), while the full sample of parents had preference for more frequent reviews. As half of the parents in the full sample had a child aged 5 or under $(n=15)$, this is not entirely surprising, as younger children need more frequent reviews due to their rapidly changing needs associated with growth and development. Interestingly, only comprehensiveness of assessment was found to be a significant attribute in all samples and thus is the most influential attribute on the service preferences of the participants in this study.

These results are congruent with the findings from previous research and recommendations from government and charitable organisation reports. Wheelchairs are important interventions for disabled children to enhance independence, social inclusion and participation [11, 32-35]. It is thus important that wheelchair provision supports optimised physical, cognitive and social development [36] and that wheelchairs are usable in all places required [25, 36, 37]. A holistic approach to assessment and performance measures should be employed to cater for the clinical, social, educational and lifestyle needs of service users $[38,39]$, which is reflected in published healthcare standards [33]. In order for disabled children to achieve the best outcomes, wheelchairs must be delivered quickly and within set timelines [15-18, 25, 36, 38, 39]. Children's needs and their wheelchairs should be reviewed at least annually [25].

From July 2015, central information about the NHS wheelchair service volume, expenditure, access and patient experience will be collected from each wheelchair service by NHS England. This will be used to build a national dataset to improve transparency and consistency across NHS wheelchair services, while meeting local supply and demand needs. Furthermore, a national wheelchair tariff designed to improve uniformity and value for money is currently being piloted before a planned national roll-out by 2017. The results from this pilot DCE study are too limited to directly inform service change in the NHS; however, the results do provide impetus for developing services that are based specifically around service user needs and preferences. This study demonstrates that DCE methods could be utilised on a larger scale to inform both national and local changes to services and potentially to develop the NHS tariff around the service preferences of children and their parents in a systematic manner. For instance, the tariff could be designed to promote provision of wheelchairs which meet clinical, social and educational needs of children.
As an example, a 2010 government report highlighted multiple issues with NHS wheelchair services in Wales, UK [17]. This report was in response to criticism from service users and charitable organisations regarding the failures of wheelchair services for children and adults in Wales, particularly waiting times and strict eligibility criteria. In 2012, the National Assembly for Wales outlined a plan for service change and provided an additional annual budget of $£ 2.2$ million to wheelchair services across Wales [40]. It is plausible that a DCE could be used to assess how Welsh wheelchair service users prioritise different attributes of these services and then assign additional funding according to service user preferences. This would enable the opinions of service users to drive investment decisions in wheelchair services. This could also be applied to current budget expenditure through service restructuring and reinvestment decisions.

However, it is difficult to see how policy decisions about service attributes could be made based solely on this type of DCE involving disabled children and their parents (even with adequate numbers and statistical power). If service commissioners were to decide to follow lean principles and strip away attributes of wheelchair services based on the results of a large scale DCE, they would be doing so based on the testimonies of families who potentially had not been exposed to aspects of the service they were being asked to state preference for. DCE data in this context would need to be supplemented with evidence of effect to see if additional service attributes improve age-related outcomes. At present, this data is limited, and thus additional research into many aspects of wheelchair provision for disabled children is needed.

Other methods of preference elicitation and service user engagement should also be considered. For instance, qualitative interviews or focus groups would likely be a simpler method of understanding the views of service users and would yield results with more validity in this context. However, qualitative methods are time consuming when gathering large quantities of data and more difficult to synthesise across the respondents. A well-designed DCE can be completed by all capable service users, and thus broader understanding of preferences across all service users may be achievable. DCEs also offer more insight into how service users trade off different attributes of services, thus revealing the relative importance of attributes. Some service users may be unable to adequately verbalise their preferences, thus forced choice DCEs can be used to reveal preferences that individuals would otherwise be unable to communicate.

In practice, a mixture of methods should be used and triangulated to gain insight, as there are pros and cons 
to both quantitative and qualitative data. Congruence across data sources would indicate a higher degree of validity and robustness.

\section{Study limitations}

This DCE study is a pilot study with small sample sizes and was designed to illustrate the use of the DCE methodology in this particular population group. It is important to note that all participants completed the DCE questionnaire in full without error or missing data and appeared to understand the instructions given.

Due to the size of the samples and their demographic characteristics, the results are not generalisable to the wider population of disabled children who use wheelchairs and their parents. The samples were relatively self-selective; thus, the important views of disengaged or unmotivated individuals may have been missed. Furthermore, it was not possible to take into account important factors such as condition prognosis, age, cognitive ability, growth and the purpose of mobility equipment (e.g. play versus mobility). In a larger sample, it would be important to examine the impact of these factors on preferences, for instance, the different follow-up requirements of children with relatively static conditions, such as cerebral palsy, compared to children with potentially rapidly progressive conditions, such as muscular dystrophy, or children with physical and cognitive disabilities. All child participants had the cognitive ability to complete the questionnaire; therefore, the child sample findings are not generalisable to children with cognitive impairments. The parent sample was more diverse, with most participants $(n=19)$ representing a child who was unable to complete their own DCE questionnaire due to age and/ or cognitive ability. Although the sample size is too small to make wider assumptions about the representativeness of the findings, the application of the method illustrates that when disabled children have capacity, DCE methods can be used appropriately as a means of preference elicitation, and that in circumstances where the child does not have capacity their parent(s) may act as a suitable proxy.

Given the small sample sizes, there is a danger of child age causing aggregation to the mean by, for example, including a parent of a 2-year-old in the same analysis as a parent of an 18-year-old, although the sub-group analysis dealt with this to some extent. The age range of the child sample (11-18 years) could also be considered too vast to draw together the results and make wider conclusions, particularly with such a small sample.

The differences between the child and parent samples in terms of child age-related needs and cognitive development are also difficult to compare, and different outcomes should be expected. Making comparisons between the child and parent samples raises some interesting issues.
As a general rule, children are not expected to take full responsibility for what happens in their lives; it is up to their parents to take this responsibility, particularly for young children. It is therefore not surprising that there were differences between child and parent preferences. Conversely, in the sub-group analysis, the child and parent preferences were relatively similar and comparable, particularly in terms of $\beta$-coefficient directions. This raises some interesting questions as to whether children and parents influenced each other's preferences or whether they genuinely had a shared sense of service preference.

\section{Conclusions}

The results from this study cannot be generalised to the wider population of disabled children and parents due to the small sample sizes and unrepresentative demographic characteristics. The results indicate that for this cohort of disabled children and their parents, the most important wheelchair service attributes were comprehensiveness of wheelchair assessment and wheelchair delivery time. These results support the findings of previous wheelchair services reports and inquiries. Future research should utilise larger and more representative samples. More research is needed into the effective measurement of outcomes from wheelchair provision, particularly addressing social, education and independence needs of disabled children.

This study illustrates the use of DCE methods to examine wheelchair service preferences of disabled children (aged 11 and over) and their parents. Care must be taken to ensure that the methods are used appropriately, for instance, taking into account the layout, language and presentation of the DCE questionnaire. Consideration of methodological implications is required when comparing child and parent preferences.

\section{Additional files}

Additional file 1: DCE supplementary notes. Full list of supplementary notes provided to participants to aid completion of the DCE questionnaire. (DOCX 15.0kb)

Additional file 2: DCE fixed effects logit model. Full disclosure of DCE fixed effects logit model used to estimate preferences. (DOCX 13.4kb)

Additional file 3: Sub-group analysis. Results from conditional logistic regression model of disabled child $(n=9)$ and parent $(n=9)$ matched pairs. (DOCX 15.2kb)

\section{Abbreviations}

DCE, discrete choice experiment; MRS, marginal rate of substitution; NHS, National Health Service; PWC, powered wheelchair; UK, United Kingdom

\section{Acknowledgements}

We would like to acknowledge the work of Nina Evans for assisting in the design of the DCE and the recruitment of participants. We would also like acknowledge the support of Carol McCudden, Fiona McNaught, Amanda Hopkin and Mark Lovell for aiding in the recruitment of participants and Emma Bray for assisting in editing and proofreading the manuscript. We 
would like to thank our wheelchair service partners for their support in recruitment and data collection throughout the study.

\section{Funding}

The study was funded by the National Institute for Social Care and Health Research as part of a PhD studentship award. The funding organisation had no role in the design, conduct, analysis or interpretation of this research.

\section{Availability of data and materials}

Data and supporting materials are available upon request.

\section{Authors' contributions}

NB, JN, RTE and NH were responsible for the study conception/design and the development of the study protocol. NB was responsible for all major study tasks, which included setting up the study, designing the DCE and all other study documentation, applying for ethical approval, engaging with the study sites, collecting the data, analysing/interpreting the results and writing the manuscript. JN, RTE and NH provided feedback on study design and documentation. JN provided expertise and guidance on the ethical application process. RTE and STY provided expertise and guidance on the DCE design. STY provided expertise and guidance on analysing the data and interpreting the results. All authors were involved in reading, revising and approving the manuscript.

\section{Authors' information}

NB is a Research Officer at the Centre for Health Economics and Medicines Evaluation (CHEME), Bangor University. NB's PhD research focussed on applying health economics methods to paediatric wheelchair provision. STY is a Research Fellow in public health economics at CHEME, Bangor University, and has published DCE research regarding diabetic retinopathy services. JN has experience in child health research, health services research with embedded health economics and evidence synthesis. $\mathrm{NH}$ is a Clinical Scientist and Director of the Designability. Recent research projects include the use of assistive technology to support home-based rehabilitation following stroke and monitoring patterns of physical activity during rehabilitation. RTE is Professor of Health Economics at CHEME, Bangor University. RTE has expertise in the economic evaluation alongside trials of complex public health and psychosocial interventions and an interest in the health economics of disability.

\section{Competing interests}

The authors declare that they have no competing interests.

\section{Consent for publication}

Not applicable.

\section{Ethics approval and consent to participate}

The study was granted ethical approval by the North West Wales NHS Research Ethics Committee (reference: 13/WA/0143) and an academic ethics committee at Bangor University. The study conformed to the tenets of the Declaration of Helsinki. Written informed consent was obtained from all participants prior to participation in the study.

\section{Author details}

${ }^{1}$ Centre for Health Economics and Medicines Evaluation, Bangor University, Ardudwy, Normal Site, Bangor, Gwynedd LL57 2PZ, UK. ${ }^{2}$ School of Social Sciences, Bangor University, Neuadd Ogwen, Bangor, Gwynedd LL57 2DG, UK. ${ }^{3}$ DesignAbility, Bath Institute of Medical Engineering, The Wolfson Centre, Royal United Hospital, Bath BA1 3NG, UK.

Received: 10 September 2015 Accepted: 25 June 2016

Published online: 19 July 2016

\section{References}

1. World Health Organization. World report on disability. Geneva: World Health Organization; 2011.

2. Sheldon S, Jacobs NA, editors. Report of a consensus conference on wheelchairs for developing countries; 2006 Nov 6-11; Bangalore, India. Copenhagen: International Society for Prosthetics and Orthotics; 2006.
3. Nations $U$. The standard rules on the equalization of opportunities for persons with disabilities: preconditions for equal participation. New York: United Nations; 1993.

4. World Health Organization. Guidelines on the provision of manual wheelchairs in less-resourced settings. Geneva: World Health Organization; 2008.

5. Contact a Family. Annual review: how I made a difference in 2010/2011. London: Contact a Family; 2011.

6. Whizz-Kidz. All Party Parliamentary Group for Paediatric Reform and WhizzKidz: "My wheelchair is my shoes". Making the case for wheelchair reform. London: Whizz-Kidz; 2011.

7. Jones MA, McEwen IR, Hansen L. Use of power mobility for a young child with spinal muscular atrophy. Phys Ther. 2003;83(3):253-62.

8. Guerette P, Furumasu J, Tefft D. The positive effects of early powered mobility on children's psychosocial and play skills. Assist Technol. 2013;25(1):39-48.

9. Butler C, Okamoto GA, McKay TM. Powered mobility for very young disabled children. Dev Med Child Neurol. 1983;25:472-4.

10. Jones MA, McEwan IR, Neas BR. Effects of power wheelchairs on the development and function of young children with severe motor impairment. Pediatr Phys Ther. 2012;24(2):131-40.

11. Wiart L, Darrah J, Hollis V, Cook A, May L. Mothers' perceptions of their children's use of powered mobility. Phys Occup Ther Pediatr. 2004;24(4):3-21.

12. Terzi L. The social model of disability: a philosophical critique. J Appl Philos. 2004;21(2):142-57.

13. Lang R. The development and critique of the social model of disability. London: Leonard Cheshire Disability and Inclusive Development Centre; 2007.

14. Audit Commission. Fully equipped 2002-assisting independence. Wetherby: Audit Commission Publications; 2002

15. NHS Modernisation Agency. Improving services for wheelchair users and carers_-good practice guide. London: Department of Health; 2005.

16. Prime Minister's Strategy Unit Report. IImproving the life chances of disabled people.' Joint report with Department for Work and Pensions, Health, Education and Skills and the Office of the Deputy Prime Minister. London: Department for Work and Pensions, Health, Education and Skills; 2005.

17. National Assembly for Wales. National assembly for Wales Health, Wellbeing and Local Government Committee: report on inquiry into wheelchair services in Wales. Cardiff: National Assembly for Wales; 2010.

18. HM Treasury and Department for Education and Skills. Aiming high for disabled children: better support for families. London/Runcorn: HM Treasury/Department for Education and Skills; 2007.

19. Bray N, Noyes J, Edwards RT, Harris N. Wheelchair interventions, services and provision for disabled children: a mixed-method systematic review and conceptual framework. BMC Health Serv Res. 2014;14:309.

20. Gidman W, Elliott R, Payne K, Meakin GH, Moore J. A comparison of parents and pediatric anesthesiologists' preferences for attributes of child daycase surgery: a discrete choice experiment. Pediatr Anesth. 2007;17:1043-52.

21. Bridges JFP, Hauber AB, Marshall D, Lloyd A, Prosser L, Regier D, Johnson FR, Mauskopf J. A checklist for conjoint analysis applications in health: report of the ISPOR conjoint analysis good research practices task force. Value Health. 2011; 14(4):403-13.

22. Curtis L. Unit costs of health and social care 2013. Cantebury: Personal Social Services Research Unit, University of Kent; 2013.

23. Sanderson D, Place M, Wright D. Evaluation of the powered wheelchair and voucher scheme initiatives. London: NHS Executive and Department of Health; 2000.

24. Muscular Dystrophy Campaign. Building on foundations: get moving - the case for effective wheelchair services. London: Muscular Dystrophy Campaign; 2010.

25. Welsh Assembly Government. National service framework for children, young people and maternity services in Wales. Cardiff: Welsh Assembly Government; 2005.

26. Sloane, NJA [Internet]. A library of orthogonal arrays [M.A.8.2.4.4.1.]. 2010 November [cited 2015 April 29]. Available from: http://neilsloane.com/oadir/ Accessed 11 Jul 2016.

27. Ryan M, Gerard K. Using discrete choice experiments to value health care programmes: current practice and future research reflections. Appl Health Econ Health Policy. 2003;2(1):55-64.

28. Ryan M, Gerard K, Amaya-Amaya M, editors. Using discrete choice experiments to value health and health and care. Netherlands: Springer; 2008.

29. Fiebig DG, Louviere $\mathrm{J}$, Walsman DM. Contemporary issues in modelling discrete choice experimental data in health economics: working paper. Australia: School of Economics, University of New South Wales; 2005. 
30. Green CJ, Gerard K. Exploring the social of health-care interventions: a stated preference discrete choice experiment. Health Econ. 2009;18(8):951-76.

31. Yeo ST, Edwards RT, Fargher EA, Luzio SD, Thomas RL, Owens DR. Preferences of people with diabetes for diabetic retinopathy screening: a discrete choice experiment. Diabet Med. 2012;29(7):869-77.

32. Evans S, Neophytou C, De Souza L, Frank AO. Young people's experiences of using electric powered indoor-outdoor wheelchairs (EPIOCs): potential for enhancing users' development? Disabil Rehabil. 2007;29(16):1281-94.

33. Wiart L, Darrah J, Cook A, Hollis V, May L. Evaluation of powered mobility use in home and community environments. Phys Occup Ther Pediatr. 2003; 23(2):59-75.

34. Home AM, Ham R. Provision of powered mobility equipment to young children: the Whizz-Kidz experience. Int J Ther Rehabil. 2003;10(11):511-9.

35. Bottos M, Bolcati C, Sciuto L, Ruggeri C, Feliciangeli A. Powered wheelchairs and independence in young children with tetraplegia. Dev Med Child Neurol. 2001;43(11):769-77.

36. Department of Health. Standard 8 of the Children's National Service Framework: disabled children and those with complex healthcare needs. London: Department of Health; 2004.

37. Care Services Improvement Partnership. Out and about—wheelchairs as part of a whole-systems approach to independence. London: Department of Health; 2006

38. Department of Health Commissioning Team. Local innovation in wheelchair and seating services. London: Department of Health; 2010.

39. National Wheelchair Managers Forum. Healthcare standards for NHSCommissioned Wheelchair Services May 2010. London: National Wheelchair Managers Forum; 2010.

40. National Assembly for Wales. Wheelchair services in Wales: follow-up inquiry. Cardiff: National Assembly for Wales; 2012.

\section{Submit your next manuscript to BioMed Central and we will help you at every step:}

- We accept pre-submission inquiries

- Our selector tool helps you to find the most relevant journal

- We provide round the clock customer support

- Convenient online submission

- Thorough peer review

- Inclusion in PubMed and all major indexing services

- Maximum visibility for your research

Submit your manuscript at www.biomedcentral.com/submit

\section{) Biomed Central}

